\title{
ON THE ESTIMATION OF $x^{T} A^{-1} x$ FOR SYMMETRIC MATRICES*
}

\author{
PARASKEVI FIKA ${ }^{\dagger}$, MARILENA MITROULI $^{\dagger}$, AND ONDŘEJ TUREK ${ }^{\ddagger}$
}

\begin{abstract}
The central mathematical problem studied in this work is the estimation of the quadratic form $x^{T} A^{-1} x$ for a given symmetric positive definite matrix $A \in \mathbb{R}^{n \times n}$ and vector $x \in \mathbb{R}^{n}$. Several methods to estimate $x^{T} A^{-1} x$ without computing the matrix inverse are proposed. The precision of the estimates is analyzed both analytically and numerically.
\end{abstract}

Key words. Quadratic forms, Matrix inverse, Kantorovich inequality, Estimates, Upper bounds.

AMS subject classifications. 15A63, 15A45, 15A18.

1. Introduction. Let $A$ be a symmetric positive definite matrix of order $n$ and $x \in \mathbb{R}^{n}$. The subject of this work is to estimate the quadratic form $x^{T} A^{-1} x$ without the direct computation of the matrix $A^{-1}$. The motivation behind our approach is to avoid the expensive evaluation of a matrix inverse that results in $\mathcal{O}\left(n^{3}\right)$ required for floating point operations. Furthermore, for special choices of the vector $x$, we can estimate useful quantities arising frequently in many applications. For instance, by choosing appropriate columns of the identity matrix for vector $x$, estimates for the diagonal entries of $A^{-1}$ are developed.

The quality of the proposed estimates for $x^{T} A^{-1} x$ will be assessed by specifying upper bounds for the absolute error of the estimation. A crucial role in deriving these bounds is played by the Kantorovich inequality (cf. [6, Formula 7.4.12.1], [7])

$$
\frac{\left(x^{T} x\right)^{2}}{\left(x^{T} A x\right)\left(x^{T} A^{-1} x\right)} \geq \frac{4 \lambda_{\min } \lambda_{\max }}{\left(\lambda_{\min }+\lambda_{\max }\right)^{2}},
$$

where $\lambda_{\min }$ and $\lambda_{\max }$ denote the minimum and the maximum eigenvalues of the matrix $A$, respectively.

We will express some of the bounds for the error of the estimation of $x^{T} A^{-1} x$ in terms of the index of proximity, which is defined for a given symmetric positive definite matrix $A$ of order $n$ and a vector $x$ as

$$
\rho(x)=\frac{\|x\|^{2} x^{T} A^{2} x}{\left(x^{T} A x\right)^{2}} .
$$

The index of proximity can be regarded as an easily computable measure of closeness of $x$ to an eigenvector of $A$, in accordance with the following observation.

OBSERVATION 1.1. Let $A \in \mathbb{R}^{n \times n}$ be a positive definite matrix and $x \in \mathbb{R}^{n}$ be a nonzero vector.

Then $\rho(x) \geq 1$. Moreover, $\rho(x)=1$ if and only if $x$ is an eigenvector of $A$.

\footnotetext{
*Received by the editors on August 20, 2020. Accepted for publication on June 30, 2021. Handling Editor: Valeria Simoncini. Corresponding Author: Marilena Mitrouli

$\dagger$ Department of Mathematics, National and Kapodistrian University of Athens, Panepistimiopolis, 15784 Athens, Greece (pfika@math.uoa.gr, mmitroul@math.uoa.gr).

${ }^{\ddagger}$ Department of Mathematics, University of Ostrava, 70103 Ostrava, Czech Republic (ondrej.turek@osu.cz).
} 
Proof. The inequality $\rho(x) \geq 1$ follows from the Cauchy-Schwarz inequality $\left(x^{T} A x\right)^{2} \leq\left(x^{T} x\right)\left(x^{T} A^{2} x\right)$. Furthermore, we have

$$
\rho(x)=1 \quad \Leftrightarrow \quad \frac{\|x\|^{2} \cdot\|A x\|^{2}}{\left(x^{T} A x\right)^{2}}=1 \quad \Leftrightarrow \quad\left|x^{T} A x\right|=\|x\| \cdot\|A x\| .
$$

By the Cauchy-Schwarz inequality, $\left|x^{T} A x\right|=\|x\| \cdot\|A x\|$ if and only if the vectors $x$ and $A x$ are linearly dependent, i.e., $A x=\lambda x$ for some $\lambda \in \mathbb{R}$. Hence, $\rho(x)=1$ iff $x$ is an eigenvector of $A$.

The paper is structured as follows: Section 2 overviews an appropriate vector decomposition that forms the base for deriving the estimates. In Section 3, we present explicit estimates for $x^{T} A^{-1} x$ and upper bounds for the related absolute error. Section 4 describes how the results of Section 3 can be applied for the estimation of the bilinear form $x^{T} A^{-1} y$. Section 5 reports numerical examples that illustrate the performance of the proposed estimates. Finally, conclusions are drawn in Section 6.

Throughout the paper $\|\cdot\|$ is the 2-norm, the superscript $T$ denotes the transpose, $\kappa$ is the condition number of a matrix, and the vector $\delta_{i}$ stands for the $i$ th column of the identity matrix. For the sake of brevity, the index of proximity $\rho(x)$ will be usually denoted simply as $\rho$.

2. Overview of the method. Let $A \in \mathbb{R}^{n \times n}$ be a positive definite symmetric matrix and $x \in \mathbb{R}^{n}$. We start from a decomposition of the vector $x$ in the form

$$
x=a-b,
$$

where $a$ is a vector parallel to $A x$, so $a=\alpha A x$ for an $\alpha>0$, and the vector $b$ represents the residual part. That is,

$$
x=\alpha A x-b .
$$

The decomposition (2.3) implies

$$
A^{-1} x=\alpha x-A^{-1} b
$$

hence

$$
x^{T} A^{-1} x=\alpha\|x\|^{2}-x^{T} A^{-1} b .
$$

The term $\alpha\|x\|^{2}$ stands for the estimate of $x^{T} A^{-1} x$, and $x^{T} A^{-1} b$ is the difference between the estimate and the true value. The absolute value of $x^{T} A^{-1} b$ will be denoted as $E_{a b s}$ and is actually the absolute error.

The aforementioned ideas can be inverted. Consider an estimation

$$
x^{T} A^{-1} x \approx E s t
$$

where the value Est is in principle arbitrary. Setting

$$
\alpha=\frac{E s t}{\|x\|^{2}},
$$

and

$$
b=\alpha A x-x,
$$

the quadratic form $x^{T} A^{-1} x$ obviously satisfies

$$
x^{T} A^{-1} x=E s t-x^{T} A^{-1} b,
$$

so the error of the estimate (2.5) - the difference between the estimate and the true value - can be again expressed as a scalar product $x^{T} A^{-1} b$ : 


$$
\text { Est }-x^{T} A^{-1} x=x^{T} A^{-1} b,
$$

where $b$ is given by (2.7).

Let us now focus on the error term $x^{T} A^{-1} b$ appearing in formulas (2.4) and (2.8). In Proposition 2.1, we derive upper bounds on the error in case of a general estimate $x^{T} A^{-1} x \approx \alpha\|x\|^{2}$. More specific versions of the bounds, adapted for particular choices of the decomposition (2.3) or for specifically chosen $\alpha$, will be derived later in forthcoming sections.

Proposition 2.1. Let $A \in \mathbb{R}^{n \times n}$ be a positive definite symmetric matrix and $x \in \mathbb{R}^{n}$. Then the absolute error of the approximation $\left(x^{T} A^{-1} x\right) \approx \alpha\|x\|^{2}$ obeys the following upper bounds.

$$
E_{a b s}=\left|\alpha\|x\|^{2}-x^{T} A^{-1} x\right| \leq \begin{cases}\frac{1+\kappa^{2}}{2 \kappa} \cdot \frac{\|x\|^{2}}{\|A x\|} \cdot\|b\| & \text { (Upper Bound I); } \\ \frac{1+\kappa^{2}}{2 \kappa} \cdot \frac{\|b\|^{2}}{\|A b\|} \cdot\|x\| & \text { (Upper Bound II); } \\ \frac{(1+\kappa)^{2}}{4 \kappa} \cdot \frac{\|x\|^{2}}{\sqrt{x^{T} A x}} \cdot \frac{\|b\|^{2}}{\sqrt{b^{T} A b}} & \text { (Upper Bound III) },\end{cases}
$$

where $b=\alpha A x-x$, the symbol $\lambda_{\min }$ denotes the minimum eigenvalue of $A$, and $\kappa=\frac{\lambda_{\max }}{\lambda_{\min }}$ is the condition number of $A$.

Proof. For estimate Est $=\alpha\|x\|^{2}$, the absolute error is given by (2.9) as

$$
E_{a b s}=\left|\alpha\|x\|^{2}-x^{T} A^{-1} x\right|=\left|x^{T} A^{-1} b\right|,
$$

where $b=\alpha A x-x$. Let us now find bounds on $\left|x^{T} A^{-1} b\right|$.

Upper Bound I. Since $A$ is symmetric, and so is $A^{-1}$, we have

$$
\left|x^{T} A^{-1} b\right|=\left|\left(A^{-1} x\right)^{T} b\right| \leq\left\|A^{-1} x\right\| \cdot\|b\| .
$$

A bound on the quantity $\left\|A^{-1} x\right\|$ can be obtained by using the Kantorovich inequality as follows. Since $A^{2}$ is a symmetric positive definite matrix, we have

$$
\frac{\left(x^{T} x\right)^{2}}{\left(x^{T} A^{2} x\right)\left(x^{T}\left(A^{2}\right)^{-1} x\right)} \geq \frac{4 \lambda_{\min }\left(A^{2}\right) \lambda_{\max }\left(A^{2}\right)}{\left(\lambda_{\min }\left(A^{2}\right)+\lambda_{\max }\left(A^{2}\right)\right)^{2}} .
$$

Obviously, $\lambda_{\min }\left(A^{2}\right)=\lambda_{\min }^{2}$ and $\lambda_{\max }\left(A^{2}\right)=\lambda_{\max }^{2}$, where $\lambda_{\min }$ and $\lambda_{\max }$ are the minimum and the maximum eigenvalues of the matrix $A$, respectively. Thus,

$$
\frac{\left(x^{T} x\right)^{2}}{\left(x^{T} A^{2} x\right)\left(x^{T} A^{-2} x\right)} \geq \frac{4 \lambda_{\min }^{2} \lambda_{\max }^{2}}{\left(\lambda_{\min }^{2}+\lambda_{\max }^{2}\right)^{2}}
$$

and so

$$
x^{T} A^{-2} x \leq \frac{\|x\|^{4}}{x^{T} A^{2} x} \frac{\left(\lambda_{\min }^{2}+\lambda_{\max }^{2}\right)^{2}}{4 \lambda_{\min }^{2} \lambda_{\max }^{2}} .
$$

Hence, we obtain

$$
\left\|A^{-1} x\right\|=\sqrt{\left(A^{-1} x\right)^{T} A^{-1} x}=\sqrt{x^{T} A^{-2} x} \leq \sqrt{\frac{\|x\|^{4}}{x^{T} A^{2} x} \frac{\left(\lambda_{\min }^{2}+\lambda_{\max }^{2}\right)^{2}}{4 \lambda_{\min }^{2} \lambda_{\max }^{2}}}=\frac{\|x\|^{2}}{\sqrt{x^{T} A^{2} x}} \frac{\lambda_{\min }^{2}+\lambda_{\max }^{2}}{2 \lambda_{\min } \lambda_{\max }} .
$$


Noting that $x^{T} A^{2} x=(A x)^{T} A x=\|A x\|^{2}$ and

$$
\frac{\lambda_{\min }^{2}+\lambda_{\max }^{2}}{2 \lambda_{\min } \lambda_{\max }}=\frac{1+\kappa^{2}}{2 \kappa},
$$

we conclude that

$$
\left\|A^{-1} x\right\| \leq \frac{\|x\|^{2}}{\|A x\|} \cdot \frac{1+\kappa^{2}}{2 \kappa} .
$$

To sum up,

$$
\left|\left(A^{-1} x\right)^{T} b\right| \leq\left\|A^{-1} x\right\| \cdot\|b\| \leq \frac{\|x\|^{2}}{\|A x\|} \cdot \frac{1+\kappa^{2}}{2 \kappa} \cdot\|b\| .
$$

Upper Bound II. Similarly as above, applying the Cauchy-Schwarz inequality

$$
\left|x^{T} A^{-1} b\right| \leq\|x\| \cdot\left\|A^{-1} b\right\|,
$$

together with Kantorovich inequality

$$
\left\|A^{-1} b\right\| \leq \frac{\|b\|^{2}}{\sqrt{b^{T} A^{2} b}} \cdot \frac{1+\kappa^{2}}{2 \kappa}=\frac{\|b\|^{2}}{\|A b\|} \cdot \frac{1+\kappa^{2}}{2 \kappa},
$$

we get

$$
\left|x^{T} A^{-1} b\right| \leq\|x\| \cdot \frac{\|b\|^{2}}{\|A b\|} \cdot \frac{1+\kappa^{2}}{2 \kappa} .
$$

Upper Bound III. We have

$$
\left|x^{T} A^{-1} b\right|=\left|\left(A^{-1 / 2} x\right)^{T} A^{-1 / 2} b\right| \leq\left\|A^{-1 / 2} x\right\| \cdot\left\|A^{-1 / 2} b\right\|=\sqrt{\left(x^{T} A^{-1} x\right) \cdot\left(b^{T} A^{-1} b\right)} .
$$

The Kantorovich inequality gives the bounds

$$
x^{T} A^{-1} x \leq \frac{\|x\|^{4}}{x^{T} A x} \cdot \frac{(1+\kappa)^{2}}{4 \kappa} \quad \text { and } \quad b^{T} A^{-1} b \leq \frac{\|b\|^{4}}{b^{T} A b} \cdot \frac{(1+\kappa)^{2}}{4 \kappa} ;
$$

hence

$$
\left|x^{T} A^{-1} b\right| \leq \sqrt{\frac{\|x\|^{4}}{x^{T} A x} \cdot \frac{(1+\kappa)^{2}}{4 \kappa} \cdot \frac{\|b\|^{4}}{b^{T} A b} \cdot \frac{(1+\kappa)^{2}}{4 \kappa}}=\frac{\|x\|^{2}}{\sqrt{x^{T} A x}} \cdot \frac{\|b\|^{2}}{\sqrt{b^{T} A b}} \cdot \frac{(1+\kappa)^{2}}{4 \kappa} .
$$

3. Estimation of $x^{T} A^{-1} x$. In this section, we introduce explicit estimates for the quadratic form $x^{T} A^{-1} x$. They will be obtained in two different manners: by minimizing the upper bound on the error and heuristically from certain identities valid for vectors with property $\rho(x)=1$, that is, for eigenvectors of $A$. Efficient explicit bounds on the error will be also derived.

3.1. Minimization of the bounds on the error. The idea consists in searching for the value $\alpha$ such that the decomposition (2.3) minimizes some of the upper bounds on the absolute error $E_{a b s}=\left|x^{T} A^{-1} b\right|$ that were formulated in Proposition 2.1. Recall that for a chosen $\alpha$, the residual part $b$ is given by equation (2.7) as $b=\alpha A x-x$.

In forthcoming paragraphs, we will consider three of the upper bounds of the absolute error $\left|x^{T} A^{-1} b\right|$ presented in Proposition 2.1, namely, Upper Bound I, Upper Bound II, and Upper Bound III. 
Upper Bound I. Let us start with Upper Bound I, i.e.,

$$
E_{a b s} \leq \frac{1+\kappa^{2}}{2 \kappa} \cdot \frac{\|x\|^{2}}{\|A x\|} \cdot\|b\| .
$$

The smaller $\|b\|$ is, the smaller the quantity on the right-hand side is. With regard to equation (2.7), the norm of $b$ becomes minimal for $b$ being perpendicular to $A x$. Then equation (2.3) gives

$$
x^{T} A x=(\alpha A x-b)^{T} A x=\alpha(A x)^{T} A x-b^{T} A x=\alpha\|A x\|^{2}-0 ;
$$

hence

$$
\alpha=\frac{x^{T} A x}{\|A x\|^{2}} .
$$

In this way, we have obtained the estimate

$$
x^{T} A^{-1} x \approx \alpha\|x\|^{2}=\frac{x^{T} A x}{\|A x\|^{2}}\|x\|^{2}
$$

let us denote it Est1.

REMARK 3.1. This estimate can be obtained also via the extrapolation method shown in [4]. The present derivation elucidates its connection with error minimization. Therefore, it allows to express an upper bound for the absolute error, which is not possible through the extrapolation procedure of [4].

Let us proceed to finding an explicit upper bound on the error term. Equation (2.3) gives

$$
b=\alpha A x-x=\frac{x^{T} A x}{\|A x\|^{2}} A x-x .
$$

Then the orthogonality of vectors $A x, b$ together with the Pythagorean theorem implies

$$
\|b\|=\|x\| \sqrt{1-\frac{\left(x^{T} A x\right)^{2}}{\|x\|^{2}\|A x\|^{2}}}=\|x\| \sqrt{1-\frac{1}{\rho}} .
$$

Combining this result with (3.11), we arrive at the following bound on the error:

$$
E_{a b s} \leq \frac{1+\kappa^{2}}{2 \kappa} \cdot \sqrt{1-\frac{1}{\rho}} \cdot \frac{\|x\|^{3}}{\|A x\|} .
$$

Let us note that Proposition 2.1 allows to find other explicit expressions for the upper bound on the error of estimate Est1.

Upper Bound II. Now we will derive an estimate that minimizes Upper Bound II, which reads

$$
E_{a b s} \leq \frac{1+\kappa^{2}}{2 \kappa} \cdot\|x\| \cdot \frac{\|b\|^{2}}{\|A b\|} .
$$

The quantity on the right-hand side is minimized for $\frac{\|b\|^{2}}{\|A b\|}$ being as small as possible.

Using (2.7), we obtain

$$
\|b\|^{2}=b^{T} b=(\alpha A x-x)^{T}(\alpha A x-x)=\alpha^{2}\left(x^{T} A^{2} x\right)-2 \alpha\left(x^{T} A x\right)+\|x\|^{2},
$$


and

$$
\|A b\|^{2}=b^{T} A^{2} b=\alpha^{2}\left(x^{T} A^{4} x\right)-2 \alpha\left(x^{T} A^{3} x\right)+x^{T} A^{2} x .
$$

For the sake of brevity, let us denote

$$
c_{k}=x^{T} A^{k} x, \quad k=0,1,2, \ldots
$$

Then

$$
\frac{\|b\|^{2}}{\|A b\|}=\frac{\alpha^{2} c_{2}-2 \alpha c_{1}+c_{0}}{\sqrt{\alpha^{2} c_{4}-2 \alpha c_{3}+c_{2}}} .
$$

Regarding this quantity as a function of a variable $\alpha$, one can find its minimum by differential calculus. The derivative is zero for

$$
2\left(\alpha c_{2}-c_{1}\right)\left(\alpha^{2} c_{4}-2 \alpha c_{3}+c_{2}\right)=\left(\alpha^{2} c_{2}-2 \alpha c_{1}+c_{0}\right)\left(\alpha c_{4}-c_{3}\right) .
$$

After a manipulation, the above equation leads to the problem of finding roots of a polynomial of degree 3 , namely,

$$
c_{2} c_{4} \alpha^{3}-3 c_{2} c_{3} \alpha^{2}+\left(2 c_{2}^{2}+2 c_{1} c_{3}-c_{0} c_{4}\right) \alpha+c_{0} c_{3}-2 c_{1} c_{2}=0 .
$$

Finding the real roots and choosing the root $\hat{\alpha}$ that minimizes (3.14), we obtain the estimate

$$
x^{T} A^{-1} x \approx \hat{\alpha}\|x\|^{2} .
$$

In what follows, this estimation will be referred to as Est2.

By construction, estimate Est2 corresponds to Upper Bound II on the error, given by

$$
E_{a b s} \leq\|x\| \cdot \frac{1+\kappa^{2}}{2 \kappa} \cdot \frac{\hat{\alpha}^{2}\left(x^{T} A^{2} x\right)-2 \hat{\alpha}\left(x^{T} A x\right)+\|x\|^{2}}{\sqrt{\hat{\alpha}^{2}\left(x^{T} A^{4} x\right)-2 \hat{\alpha}\left(x^{T} A^{3} x\right)+x^{T} A^{2} x}} .
$$

Other upper bounds on the absolute error of estimate Est2 can be obtained using Proposition 2.1.

Upper Bound III. Finally, let us explore Upper Bound III on the absolute error, i.e.,

$$
E_{a b s} \leq \frac{(1+\kappa)^{2}}{4 \kappa} \cdot \frac{\|x\|^{2}}{\sqrt{x^{T} A x}} \cdot \frac{\|b\|^{2}}{\sqrt{b^{T} A b}} .
$$

The bound becomes minimized for $\frac{\|b\|^{2}}{\sqrt{b^{T} A b}}$ being as small as possible.

We can proceed similarly as above. Applying (2.7), we get

$$
b^{T} A b=\alpha^{2}(A x)^{T} A^{2} x-2 \alpha\|A x\|^{2}+x^{T} A x=\alpha^{2} c_{3}-2 \alpha c_{2}+c_{1} .
$$

Hence,

$$
\frac{\|b\|^{2}}{\sqrt{b^{T} A b}}=\frac{\alpha^{2} c_{2}-2 \alpha c_{1}+c_{0}}{\sqrt{\alpha^{2} c_{3}-2 \alpha c_{2}+c_{1}}} .
$$

Differentiating this quantity as a function of a variable $\alpha$, we find that the derivative is zero for

$$
c_{2} c_{3} \alpha^{3}-3 c_{2}^{2} \alpha^{2}+\left(4 c_{1} c_{2}-c_{0} c_{3}\right) \alpha+c_{0} c_{2}-2 c_{1}^{2}=0 .
$$


Calculating the roots and selecting the root $\hat{\alpha}$ that minimizes (3.15), we get the estimate

$$
x^{T} A^{-1} x \approx \hat{\alpha}\|x\|^{2}
$$

which we will referred to as Est3.

Estimate Est3 features the following Upper Bound III on the absolute error:

$$
E_{a b s} \leq \frac{\|x\|^{2}}{\sqrt{x^{T} A x}} \cdot \frac{(1+\kappa)^{2}}{4 \kappa} \cdot \frac{\hat{\alpha}^{2}\left(x^{T} A^{2} x\right)-2 \hat{\alpha}\left(x^{T} A x\right)+\|x\|^{2}}{\sqrt{\hat{\alpha}^{2}\left(x^{T} A^{3} x\right)-2 \hat{\alpha}\left(x^{T} A^{2} x\right)+x^{T} A x}} .
$$

One can also apply Proposition 2.1 for getting other upper bounds (i.e., Upper Bounds I and II).

3.2. Estimates based on the index of proximity. In this section, we will present another method to construct estimates for $x^{T} A^{-1} x$ with the property of being exact if $\rho(x)=1$. It is based on the following observation.

Observation 3.2. Let $A \in \mathbb{R}^{n \times n}$ be a positive definite matrix and $x \in \mathbb{R}^{n}$ be a nonzero vector. The following statements are equivalent:

- $\rho(x)=1$;

- $\rho\left(A^{k} x\right)=1$ for any $k \in \mathbb{R}$.

Proof. By Observation 1.1, $\rho(x)=1$ iff $x$ is an eigenvector of $A$. This is further equivalent to $A^{k} x$ being an eigenvector of $A$, and so with $\rho\left(A^{k} x\right)=1$ for any $k \in \mathbb{R}$.

Let us now consider a particular value of $k$, namely, $k=-1 / 2$. The reason for this choice is that $\rho\left(A^{-1 / 2} x\right)$, when evaluated by equation (1.2), contains explicitly the quadratic form $x^{T} A^{-1} x$, which is readily seen from the derivation below:

$$
\rho\left(A^{-\frac{1}{2}} x\right)=\frac{\left\|A^{-\frac{1}{2}} x\right\|^{2} \cdot\left\|A^{\frac{1}{2}} x\right\|^{2}}{\left(\left(A^{-\frac{1}{2}} x\right)^{T} A^{\frac{1}{2}} x\right)^{2}}=\frac{\left(\left(A^{-\frac{1}{2}} x\right)^{T} A^{-\frac{1}{2}} x\right)\left(\left(A^{\frac{1}{2}} x\right)^{T} A^{\frac{1}{2}} x\right)}{\left(x^{T} x\right)^{2}}=\frac{\left(x^{T} A^{-1} x\right)\left(x^{T} A x\right)}{\|x\|^{4}} .
$$

Hence, we can express $x^{T} A^{-1} x$,

$$
x^{T} A^{-1} x=\frac{\|x\|^{4}}{x^{T} A x} \rho\left(A^{-\frac{1}{2}} x\right)
$$

In view of Observation 3.2, we can approximately replace $\rho\left(A^{-\frac{1}{2}} x\right)$ with any expression of type

$$
\rho\left(A^{-\frac{1}{2}} x\right) \approx \rho\left(A^{k_{1}} x\right)^{p_{1}} \cdots \rho\left(A^{k_{m}} x\right)^{p_{m}}
$$

where $k_{1}, \ldots, k_{m}$ are nonnegative integers and $p_{1}, \ldots, p_{m} \in \mathbb{R}$, and the approximation becomes identity for $\rho(x)=1$. In this way, one can construct a variety of estimates of $x^{T} A^{-1} x$ that are exact for $\rho(x)=1$.

For example, estimate Est1 is obtained by replacing $\rho\left(A^{-\frac{1}{2}} x\right)$ approximately with $\rho(x)^{-1}$; indeed, we have

$$
x^{T} A^{-1} x=\frac{\|x\|^{4}}{x^{T} A x} \rho\left(A^{-\frac{1}{2}} x\right) \approx \frac{\|x\|^{4}}{x^{T} A x}(\rho(x))^{-1}=\frac{\|x\|^{4}}{x^{T} A x} \cdot \frac{\left(x^{T} A x\right)^{2}}{\|x\|^{2} \cdot\|A x\|^{2}}=\frac{\|x\|^{2} x^{T} A x}{\|A x\|^{2}}=\text { Est1. }
$$

Below we will establish another estimate of $x^{T} A^{-1} x$ that suggests itself naturally. We start from the ansatz

$$
\rho\left(A^{-\frac{1}{2}} x\right) \cdot \rho\left(A^{\frac{1}{2}} x\right)=\rho(x)^{2},
$$


that is, we assume that $\rho\left(A^{0} x\right)$ is equal to the geometric mean of $\rho\left(A^{-\frac{1}{2}} x\right)$ and $\rho\left(A^{\frac{1}{2}} x\right)$. Combining (3.17) with (3.16), we obtain

$$
x^{T} A^{-1} x=\frac{\|x\|^{4}}{x^{T} A x} \rho\left(A^{-\frac{1}{2}} x\right) \approx \frac{\|x\|^{4}}{x^{T} A x} \cdot \frac{\rho(x)^{2}}{\rho\left(A^{\frac{1}{2}} x\right)},
$$

and since

$$
\begin{aligned}
\rho(x) & =\frac{\|x\|^{2} x^{T} A^{2} x}{\left(x^{T} A x\right)^{2}}=\frac{\|x\|^{2}\|A x\|^{2}}{\left(x^{T} A x\right)^{2}}, \\
\rho\left(A^{\frac{1}{2}} x\right) & =\frac{\left\|A^{\frac{1}{2}} x\right\|^{2}\left(A^{\frac{1}{2}} x\right)^{T} A^{2} A^{\frac{1}{2}} x}{\left(\left(A^{\frac{1}{2}} x\right)^{T} A A^{\frac{1}{2}} x\right)^{2}}=\frac{\left(x^{T} A x\right)\left(x^{T} A^{3} x\right)}{\|A x\|^{4}},
\end{aligned}
$$

we get

$$
x^{T} A^{-1} x \approx \frac{\|x\|^{4}}{x^{T} A x} \cdot\left(\frac{\|x\|^{2}\|A x\|^{2}}{\left(x^{T} A x\right)^{2}}\right)^{2} \cdot \frac{\|A x\|^{4}}{\left(x^{T} A x\right)\left(x^{T} A^{3} x\right)}=\frac{\|x\|^{8}\|A x\|^{8}}{\left(x^{T} A x\right)^{6}\left(x^{T} A^{3} x\right)} .
$$

From now on let us refer to the estimate

$$
x^{T} A^{-1} x \approx \frac{\|x\|^{8} \cdot\|A x\|^{8}}{\left(x^{T} A x\right)^{6} \cdot\left(x^{T} A^{3} x\right)},
$$

as to Est4. Its error can be bounded by means of Proposition 2.1, where we set

$$
\alpha=\frac{E s t 4}{\|x\|^{2}}=\frac{1}{\|x\|^{2}} \cdot \frac{\|x\|^{8} \cdot\|A x\|^{8}}{\left(x^{T} A x\right)^{6}\left(x^{T} A^{3} x\right)}=\frac{\|x\|^{6} \cdot\|A x\|^{8}}{\left(x^{T} A x\right)^{6}\left(x^{T} A^{3} x\right)}
$$

and

$$
b=\alpha A x-x=\frac{\|x\|^{6} \cdot\|A x\|^{8}}{\left(x^{T} A x\right)^{6}\left(x^{T} A^{3} x\right)} A x-x .
$$

4. Estimation of the bilinear form $x^{T} A^{-1} y$. Note that the results of Section 3 can be applied for the estimation of the bilinear form $x^{T} A^{-1} y$. This is done by the polarization identity

$$
x^{T} A^{-1} y=\frac{1}{4}\left(w^{T} A^{-1} w-v^{T} A^{-1} v\right),
$$

where $w=x+y$ and $v=x-y$. In particular, the upper and lower bounds for the quadratic forms $w^{T} A^{-1} w$ and $v^{T} A^{-1} v$ give bounds for $x^{T} A^{-1} y$.

5. Numerical Implementation. In this section, numerical experiments and comparisons for the estimation of the quadratic form $x^{T} A^{-1} x$ are presented for several positive definite symmetric matrices.

5.1. Numerical Examples. The following tables display the estimations Est2, Est3, Est4 and the lower and upper bounds for the exact value of $\left(x^{T} A^{-1} x\right)$. In most of the cases, the upper and lower bounds on $\left(x^{T} A^{-1} x\right)$ arising from a given estimate $E s t$ are calculated as $E s t+U B$ and Est-UB, respectively, for $U B$ being the corresponding minimal upper bound for the error $E_{a b s}$. In some of the test examples, the upper bounds for the error $E_{a b s}$ are reported as well. In particular, the third column of these Tables contains the 
corresponding upper bounds for the error, for each estimation. For notational simplicity, the upper bounds Upper Bound I to Upper Bound III will be denoted as UB1-UB3, respectively.

In the following examples, all computations are conducted using MATLAB codes (R2015b 64-bit (win64), on an Intel Core i5-6200U with $8 \mathrm{~GB}$ RAM at $2.3 \mathrm{GHz}$ ). We test miscellaneous matrices to examine the behavior of the derived estimates for the quadratic form $x^{T} A^{-1} x$ and the bilinear form $x^{T} A^{-1} y$. We choose the vectors $x$ and $y$ to be either columns of the identity matrix or linear combinations of them, or vectors with random entries. Most of the test matrices $A$ are obtained by using the gallery function in MATLAB. The estimates resulting from the polarization identity are denoted as Est2 PI, Est3 PI, and Est4 $P I$.

Example 1: $A=B^{T} B$, where $B=$ Parter matrix from Matlab Gallery, $n=1000, \kappa=17.8983(B=$ gallery('parter',1000))

Parter is a Cauchy and Toeplitz matrix with entries $B(i, j)=1 /(i-j+0.5)$. In Tables 1 and 2 , we test the estimates for $x^{T} A^{-1} x$, where $A=B^{T} B$, for $x=-\delta_{10}+\delta_{n}$, and a random vector $x$ whose entries are drawn from the standard normal distribution, respectively.

Example 2: $A=$ Poisson matrix from Matlab Gallery, $n=900, \kappa=388.8121$ ( $A=$ gallery('poisson', 30))

Let us consider the Poisson matrix which is symmetric, block tridiagonal (sparse), and arises from the five-point finite difference approximation of the Poisson equation in a unit square with an $m \times m$ mesh. This matrix can be also given by using the gallery function in MATLAB. The Poisson matrix is of the form $A=\operatorname{tridiag}\left(-I_{m}, T,-I_{m}\right)$, where each block $T=\operatorname{tridiag}(-1,4,-1)$ has dimension $m$. In Table 3 , we test the estimates for $x^{T} A^{-1} x$, for $x=-\delta_{1}+\frac{1}{2} \delta_{3}$ and a random vector $x$ with entries in the interval $(0,1)$.

TABLE 1

$x=-\delta_{10}+\delta_{n}, \rho=1.0188$, exact value of $x^{T} A^{-1} x=0.2219$

\begin{tabular}{|c|c|c|c|c|c|c|}
\hline \multirow{2}{*}{$\begin{array}{c}A=B^{T} B, \\
B=\text { Parter matrix }\end{array}$} & \multirow{2}{*}{$\begin{array}{c}\text { Estimated } \\
\text { value }\end{array}$} & \multicolumn{3}{|c|}{ Upper Bounds on $E_{a b s}$} & \multicolumn{2}{|c|}{ Bounds on $x^{T} A^{-1} x$} \\
\hline & & UB1 & UB2 & UB3 & Lower & Upper \\
\hline Est2 & 0.2272 & 0.3020 & 0.3834 & 0.1975 & 0.0296 & 0.4247 \\
\hline Est3 & 0.2191 & 0.2717 & 0.4004 & 0.1922 & 0.0269 & 0.4113 \\
\hline Est4 & 0.2197 & 0.2733 & 0.3980 & 0.1922 & 0.0274 & 0.4119 \\
\hline \multicolumn{5}{|c|}{ Best lower and upper bound on $x^{T} A^{-1} x$} & 0.0296 & 0.4113 \\
\hline
\end{tabular}

TABLE 2

$x=$ random vector, $\rho=1.0004$, exact value of $x^{T} A^{-1} x=100.2273$

\begin{tabular}{|c|c|c|c|}
\hline$A=B^{T} B$, & Estimated & \multicolumn{2}{|c|}{ Bounds on $x^{T} A^{-1} x$} \\
\cline { 3 - 4 }$B=$ Parter matrix & value & Lower & Upper \\
\hline Est2 & 101.3859 & 83.5054 & 119.2664 \\
Est3 & 101.2371 & 83.3527 & 119.1215 \\
Est4 & 99.6773 & 81.7518 & 117.6028 \\
\hline Best lower and upper & & \\
bound on $x^{T} A^{-1} x$ & 83.5054 & 117.6028 \\
\hline
\end{tabular}


TABLE 3

Estimates for $x^{T} A^{-1} x$, for the Poisson matrix

\begin{tabular}{|c|c|c|}
\hline \multirow{2}{*}{$A=$ Poisson matrix } & \multicolumn{2}{|c|}{ Estimated value } \\
\cline { 2 - 3 } & $x=-\delta_{1}+\frac{1}{2} \delta_{3}, \rho=1.0875$ & $x=$ random vector, $\rho=4.5325$ \\
\hline Est2 & 0.3231 & $1.8326 \mathrm{e} 2$ \\
Est3 & 0.3125 & $1.8452 \mathrm{e} 2$ \\
Est4 & 0.3462 & $5.4155 \mathrm{e} 3$ \\
\hline Exact value of $x^{T} A^{-1} x$ & 0.3490 & $8.9657 \mathrm{e} 3$ \\
\hline
\end{tabular}

TABLE 4 $x=\delta_{1}-2 \delta_{2}+\delta_{20}, \rho=1.0256$, exact value of $x^{T} A^{-1} x=3.1963$

\begin{tabular}{|c|c|c|c|c|c|c|}
\hline $\mathrm{A}=$ Heatflow matrix & Estimated & \multicolumn{6}{|c|}{ Upper Bounds on $E_{a b s}$} & \multicolumn{2}{c|}{ Bounds on $x^{T} A^{-1} x$} \\
\cline { 3 - 7 } & value & UB1 & UB2 & UB3 & Lower & Upper \\
\hline Est2 & 3.1752 & 0.7552 & 0.7621 & 0.6485 & 2.5267 & 3.8236 \\
Est3 & 3.1099 & 0.7325 & 0.7702 & 0.6429 & 2.4670 & 3.7527 \\
Est4 & 3.1943 & 0.7642 & 0.7628 & 0.6522 & 2.5422 & 3.8465 \\
\hline \multicolumn{3}{|c|}{ Best lower and upper bound on $x^{T} A^{-1} x$} & 2.5422 & 3.7527 \\
\hline
\end{tabular}

Remark: In Table 3, we notice that, for $x=$ random vector, all the estimates underestimate the form $x^{T} A^{-1} x$. This is due to the value of $\rho=4.5325$. Indeed, since the upper bounds for the error, expressed in subsection 3.1, increase as $\rho$ moves away from the unit, the estimates are becoming less satisfactory.

In the next example, we demonstrate the behaviour of the estimates for the approximation of bilinear forms by applying the polarization identity, as described in Section 4 .

Example 3: A=Heatflow matrix, $n=10^{4}, \kappa=2.5986$

Let us consider the Heatflow matrix. This matrix is symmetric, block tridiagonal (sparse), and comes from the discretization of the linear heat flow problem using the simplest implicit finite difference method. The coefficient matrix $A$ of the resulted linear system of equations is a $m^{2} \times m^{2}$ block tridiagonal matrix $A=\operatorname{tridiag}(C, D, C)$, where $D$ is a $m \times m$ tridiagonal matrix $D=\operatorname{tridiag}(-u, 1+4 u,-u), C=$ $\operatorname{diag}([-u,-u, \ldots,-u]), u=\Delta t / h^{2}, \Delta t$ is the timestep, and $h$ is the spacing interval. The Heatflow matrix $A$ is symmetric positive definite for $u>0$. We test this matrix for $u=0.2$. In Table 4 , we see the results for estimating $x^{T} A^{-1} x$, for $x=\delta_{1}-2 \delta_{2}+\delta_{20}$.

In Table 5 , we see the estimations of the bilinear form $x^{T} A^{-1} y$ for the vectors $x=\delta_{1}, y=\delta_{2}$ and for random vectors $x, y$.

5.2. Comparison with other methods. In this section, the methods of the present paper for the estimation of the quadratic form $x^{T} A^{-1} x$ are compared with some other approaches known in the literature. In particular, we consider the following two methods.

The Gauss quadrature - Lanczos method. First, the most studied method for approximating the quadratic form $x^{T} A^{-1} x$ for symmetric matrices is the Gauss quadrature - Lanczos approach. Through this method, the bilinear form is expressed as a Riemann-Stieltjes integral which is then approximated by applying Gaussian 
TABLE 5

Estimates for $x^{T} A^{-1} y$, for the Heatflow matrix

\begin{tabular}{|c|c|c|c|c|c|c|}
\hline \multirow{2}{*}{$\begin{array}{c}A=\text { Heatflow } \\
\text { matrix }\end{array}$} & \multicolumn{2}{|c|}{$\begin{array}{c}x=\delta_{1}, y=\delta_{2}, \text { exact value } x^{T} A^{-1} y=0.0659 \\
\rho(x+y)=1.0234, \rho(x-y)=1.0150\end{array}$} & \multicolumn{3}{|c|}{$\begin{array}{c}x, y \text { random, exact value } x^{T} A^{-1} y=2.4738 e 3 \\
\rho(x+y)=1.0795, \rho(x-y)=1.0492\end{array}$} \\
\hline & \multirow{2}{*}{$\begin{array}{c}\text { Estimated } \\
\text { value }\end{array}$} & \multicolumn{2}{|c|}{ Bounds on $x^{T} A^{-1} y$} & \multirow{2}{*}{$\begin{array}{c}\text { Estimated } \\
\text { value }\end{array}$} & \multicolumn{2}{c|}{ Bounds on $x^{T} A^{-1} y$} \\
\cline { 3 - 4 } & 0.0635 & -0.0342 & 0.1612 & $2.3367 \mathrm{e} 3$ & $1.6779 \mathrm{e} 3$ & $2.9956 \mathrm{e} 3$ \\
\hline Est2 PI & 0.0621 & -0.0351 & 0.1593 & $2.3043 \mathrm{e} 3$ & $1.6443 \mathrm{e} 3$ & $2.9642 \mathrm{e} 3$ \\
Est3 PI & 0.0660 & -0.0324 & 0.1644 & $2.4617 \mathrm{e} 3$ & $1.7848 \mathrm{e} 3$ & $3.1386 \mathrm{e} 3$ \\
Est4 PI & & & & & \\
\hline Best lower and upper & & & & & $1.7996 \mathrm{e} 3$ & $2.9527 \mathrm{e} 3$ \\
\hline
\end{tabular}

quadrature rules. One needs to construct a sequence of orthogonal polynomials that is realized by using the Lanczos algorithm (cf. [5]). The computational complexity of the Lanczos algorithm is of the order $\mathcal{O}\left(k n^{2}\right)$, where $k$ is the number of the employed (Lanczos) iterations. If the Gauss quadrature rule is used, the obtained estimates also serve as lower bounds for the exact value. Gauss Lobatto quadrature rule gives estimates that are upper bounds of the exact value, but it requires the knowledge of the maximum eigenvalue of the underlying matrix. Gauss Radau quadrature rule can give estimates that are either lower or upper bounds of the exact value, depending on the usage of either the minimum or the maximum eigenvalue, respectively.

The extrapolation method. Another method for the estimation of the quadratic form $x^{T} A^{-1} x$ is based on the extrapolation of the moments of the matrix $A$ (cf. [2]). In particular, the first few terms of the sequence $x^{T} A^{k} x, k \in \mathbb{N}$ are extrapolated to $x^{T} A^{-1} x$, by expressing the quadratic forms as summations. A family of estimates that depends on a parameter $\nu \in \mathbb{R}$ (cf. [4]) have been proposed. The involved computations require only one matrix-vector product, for symmetric matrices.

In the following explicit numerical examples, we compare our estimates Est3 and Est4 with the aforementioned methods. The newly proposed estimates require only 2 matrix vector products for their implementation.

Example 4: $A=$ Sample covariance matrix, $n=1000$.

Let us consider a symmetric positive definite matrix $A=\left[a_{i j}\right]$, whose entries are computed via a decaying positive definite covariance function, i.e. $a_{i i}=1+i^{a}$ and $a_{i j}=\frac{1}{|i-j|^{b}}$ for $i \neq j$ [1]. The elements of this matrix show a decaying behavior away from the main diagonal, which simulates the decreasing correlation of high-dimensional data samples in covariance matrix analysis (cf. [8] and references therein).

In Table 6 , the quadratic forms $x^{T} A^{-1} x$ are approximated by using the iterative Gauss quadrature Lanczos method and the extrapolation method, for different values of the parameter $\nu$. In particular, we test the Gauss, Gauss Radau, and Gauss Lobatto quadrature rules. The numbers of Lanczos iterations $k$ are also reported for the applied quadrature rules. For Gauss Radau and Gauss Lobatto, we use the 'exact' smallest $\left(\lambda_{\min }\right)$ and largest $\left(\lambda_{\max }\right)$ eigenvalues. These methods are compared with the best value of the estimations developed in the present work. The order of the relative errors of the estimations and their computational complexity, in terms of the required matrix vector products (mvp's), are displayed in Table 6. The Gaussian quadrature rules allow better accuracy as the number of the Lanczos iterations increase, but this is achieved at the cost of increasing computational complexity. 
TABLE 6

Comparison of methods for sample covariance matrices

\begin{tabular}{|c|c|c|c|c|}
\hline & \multicolumn{2}{|c|}{$a=1 / 2, b=1, x=\delta_{10}, \kappa=37.1945$} & \multicolumn{2}{c|}{$a=2, b=1, x=\delta_{5}, \kappa=5.9180 e 5$} \\
& $\rho=1.1838$, exact value $\left(x, A^{-1} x\right)=0.2708$ & $\rho=1.0045$, exact value $\left(x, A^{-1} x\right)=0.0387$ \\
\hline Method & Relative error & mvp's & Relative error & mvp's \\
\hline Estimates & $E s t 4, \mathcal{O}\left(10^{-2}\right)$ & 2 & $E s t 3, \mathcal{O}\left(10^{-3}\right)$ & 2 \\
\hline Extrapolation & $\nu=-1, \mathcal{O}\left(10^{-2}\right)$ & 1 & $\nu=-1, \mathcal{O}\left(10^{-4}\right)$ & 1 \\
& $\nu=0, \nu=1(E s t 1), \mathcal{O}\left(10^{-1}\right)$ & 1 & $\nu=0, \nu=1(E s t 1), \mathcal{O}\left(10^{-3}\right)$ & 1 \\
\hline Gauss & $k=2, \mathcal{O}\left(10^{-2}\right)-\mathcal{O}\left(10^{-1}\right)$ & 2 & $k=2, \mathcal{O}\left(10^{-3}\right)-\mathcal{O}\left(10^{-2}\right)$ & 2 \\
(Radau or Lobatto) & $k=10, \mathcal{O}\left(10^{-6}\right)-\mathcal{O}\left(10^{-5}\right)$ & 10 & $k=50, \mathcal{O}\left(10^{-3}\right)-\mathcal{O}\left(10^{-2}\right)$ & 50 \\
\hline
\end{tabular}

TABLE 7

Sample covariance matrix, $a=1 / 2, b=2$. Estimation of the entire diagonal of $A^{-1}$

\begin{tabular}{|c|c|c|c|c|c|c|}
\hline \multirow{2}{*}{$n$} & \multicolumn{2}{|c|}{ Est4 } & \multicolumn{2}{c|}{ Extrapolation (Est1) } & \multicolumn{2}{c|}{ Gauss quadrature rule } \\
\cline { 2 - 7 } & mre & time (s) & mre & time (s) & mre & time (s) \\
\hline 1000 & $5.9141 \mathrm{e}-4$ & 0.0523 & $1.8188 \mathrm{e}-2$ & 0.0046 & $(\mathrm{k}=2) 3.88521 \mathrm{e}-4$ & 2.2968 \\
\hline 3000 & $1.9807 \mathrm{e}-4$ & 1.0572 & $7.5561 \mathrm{e}-3$ & 0.0375 & $(\mathrm{k}=1) 3.8273 \mathrm{e}-3$ & 23.1837 \\
& & & & & $(\mathrm{k}=2) 1.3016 \mathrm{e}-4$ & 55.2502 \\
\hline 5000 & $1.1895 \mathrm{e}-4$ & 4.5469 & $4.9587 \mathrm{e}-3$ & 0.1118 & $(\mathrm{k}=1) 2.5075 \mathrm{e}-3$ & $1.2371 \mathrm{e} 2$ \\
& & & & & $(\mathrm{k}=2) 7.8177 \mathrm{e}-5$ & $2.0482 \mathrm{e} 2$ \\
\hline
\end{tabular}

Example 5: Estimation of the entire diagonal of the matrix inverse

In this example, we see the application of the estimates for the approximation of the entire diagonal of the matrix inverse. By estimating the quadratic form $x^{T} A^{-1} x$ for $x=\delta_{i}$, estimates for the $i$ th diagonal element of the matrix $A^{-1}$ are obtained. The estimation formulae proposed in the present paper are easily applicable for the approximation of the whole diagonal of the matrix inverse. In Table 7, we see the mean relative error (mre) and the execution time in seconds for the approximation of the entire diagonal of the inverse of sample covariance matrices of dimension $n=1000, n=3000$, and $n=5000$, by using the estimates Est4, Est1 and the Gaussian ones. In general, the Gaussian estimates are not well suited for this approximation because of their element-by-element implementation that leads to high execution time.

6. Conclusions. In this work, we developed estimates for the quadratic form $x^{T} A^{-1} x$. The main advantage of the derived estimates is the avoidance of the explicit computation of the matrix inverse. The proposed formulae are of low computational complexity and satisfy certain rather sharp bounds according to the value of the index of proximity. The developed approach can be applied for the estimation of the bilinear form $x^{T} A^{-1} y$ as well through the polarization identity. The presented numerical comparison shows that the newly proposed estimates outperform the others on a majority of test cases, with the estimate Est4 having a slight edge over all the others. The Gauss quadrature - Lanczos method, as an iterative method, can attain better accuracy as the number of the Lanczos iterations grows; however, this naturally increases the computational complexity. By contrast, the approach presented in this paper leads to simple and readily implemented formulae which give fair and easy-to-compute estimations. The implementation of the proposed formulae needs neither a special software nor the a priori knowledge of any characteristic of the underlying matrix. Thus, in particular for applications such as the estimation of the entire diagonal of the matrix inverse, the proposed estimates are extremely fast. Their computational cost is 2 matrix-vector products and the attained relative error is mostly of order $\mathcal{O}\left(10^{-4}\right)-\mathcal{O}\left(10^{-2}\right)$. Moreover, the approach 
can be applied also in cases where the matrix is not known explicitly, but only through matrix-vector products.

Acknowledgments. We thank the referee for useful suggestions that led to an improvement of the manuscript. This research is co-financed by Greece and the European Union (European Social Fund- ESF) through the Operational Programme 'Human Resources Development, Education and Lifelong Learning' in the context of the project 'Reinforcement of Postdoctoral Researchers - 2nd Cycle' (MIS-5033021), implemented by the State Scholarships Foundation (IKY).

\section{REFERENCES}

[1] C. Bekas, A. Curioni and I. Fedulova. Low-cost data uncertainty quantification. Concurr. Comput.: Pract. Exp., 24(8):908-920, 2012.

[2] C. Brezinski, P. Fika, and M. Mitrouli. Moments of a linear operator, with applications to the trace of the inverse of matrices and the solution of equations. Numer. Linear Algebra Appl., 19:937-953, 2012.

[3] S.W. Drury, S. Liu, C.-Y. Lu, S. Puntanen, and G. Styan. Some comments on several matrix inequalities with applications to canonical correlations: Historical background and recent developments. Sankhya: Indian J. Stat., 64, Series A, Pt.2.:453-507, 2002.

[4] P. Fika and M. Mitrouli. Estimation of the bilinear form $y^{*} f(A) x$ for Hermitian matrices. Linear Algebra Appl., 502:140$158,2016$.

[5] G.H. Golub and G. Meurant. Matrices, Moments and Quadrature with Applications. Princeton University Press, Princeton, 2010.

[6] R.A. Horn and C.R. Johnson. Matrix Analysis. Cambridge University Press, Cambridge, 1985.

[7] L.V. Kantorovich. Functional Analysis and Applied Mathematics. Translated from Russian (Kantorovich, 1948) by CD. Benster and edited by G.E. Forsythe. NBS Report no. 1509 (1101-10-5100), U.S. Dept. of Commerce, National Bureau of Standards, Los Angeles; 1952. (See pp. 106-109.)

[8] J. Tang and Y. Saad. A probing method for computing the diagonal of a matrix inverse. Numer. Linear Algebra Appl., 19:485-501, 2012.

[9] G.S. Watson, G. Alpargu, and G.P.H. Styan. Some comments on six inequalities associated with the inefficiency of ordinary least squares with one regressor. Linear Algebra Appl., 264:13-53, 1997. (Includes English translations of Bender, 1942, Frucht, 1943 and Saleme, 1942.) 